\title{
Study on Polygonum Cuspidatum Combined with Aerobic Exercise Treating Dyslipidemia
}

\author{
Li Liu \\ Sports Department, Northwest University, Xi'an 710069, China \\ 35739659@qq.com
}

Keywords: Polygonum cuspidatum; Dyslipidemia; Aerobic exercise

\begin{abstract}
In the study investigate the ameliorative effect of the extract of Polygonum Cuspidatum (PC) in combination with aerobic exercise in hyperlipidemia rat. The rats were raised by high fat diet for four weeks; meanwhile these rats were treated with PC and aerobic exercise for four weeks. The rats were executed and the blood samples were collected. The model of hyperlipidemia was then established and the model was verified. The TC, TG, LDL-C and HDL-C of the serum were detected and the concentration of SOD,GSH-Px, CAT and MDA were also measured. The results showed that PC with aerobic exercise could decrease remarkably the concentrations of LDL-C, TC and TG. The activity of SOD,GSH-Px and CAT in PC with aerobic exercise group is promoted and level of MAD is decreased dramatically. PC in combination with aerobic exercise could treat the abnormity of serum lipid and has obviously anti-lipoperoxidation effect.
\end{abstract}

\section{Introduction}

Knotweeds, clinical common herbs, are dried Polygonum Polygonum cuspidatum rhizome and roots of perennial herbs. It has long history of medicinal, with expelling wind and dampness, stasis and pain, cough and phlegm effect and it is mainly used for joint arthralgia, heat jaundice, amenorrhea, sputum, water and fire burns, or flutter injury, boils, etc [1]. Knotweed Polygonum cuspidatum extract includes vegetarian, Polygonum prime ether, stilbene compounds, including resveratrol glycosid; it also contains tannin and polysaccharide. As a kind of drug, Polygonum cuspidatum grows in abundance, with a wide range of pharmacological activity, with a therapeutic effect on multiple human diseases systems [2]. Modern research shows that knotweed contains many active ingredients and has wide range of pharmacological effects, including antibacterial and antiviral, dilating blood vessels to lower blood pressure, improving microcirculation anti-shock, anti-thrombotic, anti-tumor, regulating metabolism. Polygonum cuspidatum utility has been universally recognized by the medical community, particularly in cardiovascular disease and cancer treatment [3]. This study intends to investigate animal experiments Polygonum cuspidatum extract combined effect of aerobic exercise intervention on experimental rat dyslipidemia lipid metabolism disorders.

\section{Materials and Methods}

Experimental Subject. 40 male SD mice, weighing from 220-280( offered by the Experimental Animal Center of the Medicine College of Xi'an Jiaotong University), animal feed purchased simultaneously. The experiment begins after one week's feeding. Take Polygonum Cuspidatum extract (Chinese Herbal Medicine, Nantong Mitsukoshi Ltd.), raw herbs 160 g, add boiling water 3 times, combined boiling liquid filtration, vacuum distillation, and concentrated to $80 \mathrm{~mL}$, namely native drug content was $2 \mathrm{~g} \bullet \mathrm{mL}-1$, as a stock solution of aqueous extract of Polygonum cuspidatum. According to the features of ISMR, inspired by the metasynthesis, the ways and means of achieving comprehensive and integrated are concluded as follow.

Experimental Design. Divide the mice into five groups at random and each group is made up of eight mice, normal groups; normal diets fed; dyslipidemia groups; high fat diet; knotweeds group. Fed 
high-fat diet while Polygonum cuspidatum extract $10 \mathrm{~g}$ crude drug / $\mathrm{kg} \bullet \mathrm{d}$; aerobic exercise group: high-fat diet at the same time forced to swim training; knotweed combined with aerobic exercise group: high-fat diet fed while ethanol extract of Polygonum cuspidatum material $10 \mathrm{~g}$ crude drug / $\mathrm{kg}$ - d, at the same time forced to swim training.

Sampling Methods. The swimming training pool is round, with smooth wall, diameter $45 \mathrm{~cm}$, depth $70 \mathrm{~cm}$, temperature $30 \sim 35{ }^{\circ} \mathrm{C}$. Rats will swim for 2 hours per day under no load conditions, except Saturdays and Sundays, a total of four weeks.In order to measure the biochemical indicators, eye venous plexus blood will be given to the mice on the first day after the experiment commenced and the end of the second week. The experiment ends at the fourth week and the mice will be given the abdominal aortic blood, , $4{ }^{\circ} \mathrm{C}, 3000 \mathrm{r} / \mathrm{min}$ centrifugation $10 \mathrm{~min}$, take the upper serum. Index like TC, TG, HDLC and LDL-C will be measured by Olympus automatic biochemical analyzer; serum glutathione like GSHPx, SOD, MDA and catalase ( CAT) will be measured by kits.

Statistical Analysis. All data from statistical analysis will be expressed in $M \pm S D$, use the t test to compare the difference between the two groups, $\mathrm{P}<0.05$ indicates a statistically significant; $\mathrm{P}<0.01$ indicates a very significant difference; $\mathrm{P}<0.001$ indicates a highly significant difference.

\section{Experimental Result}

The comparison of serum TC, TG, LDL-C, HDL-C in different time periods is shown in Table 1. Experimental results show that knotweed, Polygonum combined with aerobic exercise can effectively suppress the increase of serum TC, TG and LDL-C levels of high cholesterol diet rat and raise HDL-C levels, effects of knotweed combined with aerobic exercise is better than pure knotweed. After 4 weeks' experiment, serum Serum SOD, GSH-Px, CAT and MDA changes are shown in Table 2. The results showed that, Polygonum cuspidatum combined with aerobic exercise group and the group intervention can significantly reduce serum MDA levels, but the effect of aerobic exercise alone is not significant.

Table1 Different groups in the comparison of serum TC, TG, LDL-C, HDL-C in different time periods

\begin{tabular}{|c|c|c|c|c|c|c|}
\hline Index & Time & Control & Model control & PC & $\begin{array}{l}\text { Aerobic } \\
\text { exercise }\end{array}$ & $\begin{array}{c}\text { PC+ Aerobic } \\
\text { exercise }\end{array}$ \\
\hline \multirow{3}{*}{ TC } & 0 Weeks & $2.29 \pm 0.02$ & $2.32 \pm 0.11$ & $2.13 \pm 0.11$ & $2.21 \pm 0.18$ & $2.20 \pm 0.11$ \\
\hline & 2Weeks & $2.31 \pm 0.17$ & $3.42 \pm 0.21^{* *}$ & $2.91 \pm 0.11^{\# \#}$ & $3.28 \pm 0.21^{\#}$ & $2.88 \pm 0.22^{\# \#}$ \\
\hline & 4Weeks & $2.32 \pm 0.13$ & $4.89 \pm 0.16^{* * *}$ & $4.33 \pm 0.26^{\# \# \# ~}$ & $4.56 \pm 0.33^{\#}$ & $3.67 \pm 0.23^{\# \# \# ~}$ \\
\hline \multirow{3}{*}{ TG } & 0 Weeks & $0.58 \pm 0.01$ & $0.60 \pm 0.01$ & $0.52 \pm 0.04$ & $0.49 \pm 0.01$ & $0.59 \pm 0.03$ \\
\hline & 2Weeks & $0.61 \pm 0.06$ & $0.79 \pm 0.02^{* *}$ & $0.61 \pm 0.03^{\#}$ & $0.68 \pm 0.01$ & $0.59 \pm 0.02^{\#}$ \\
\hline & 4Weeks & $0.55 \pm 0.02$ & $1.12 \pm 0.13^{* * *}$ & $0.75 \pm 0.01^{\# \#}$ & $0.81 \pm 0.02^{\#}$ & $0.66 \pm 0.01^{\# \#}$ \\
\hline \multirow{3}{*}{ LDL-C } & 0 Weeks & $1.03 \pm 0.21$ & $1.15 \pm 0.23$ & $0.99 \pm 0.05$ & $1.16 \pm 0.13$ & $0.97 \pm 0.03$ \\
\hline & 2Weeks & $0.97 \pm 0.01$ & $0.72 \pm 0.03^{* *}$ & $0.81 \pm 0.03$ & $0.79 \pm 0.31$ & $0.83 \pm 0.14^{\#}$ \\
\hline & 4Weeks & $0.98 \pm 0.03$ & $0.61 \pm 0.01^{* * *}$ & $0.75 \pm 0.01^{\# \# \# ~}$ & $0.71 \pm 0.03^{\#}$ & $0.84 \pm 0.21^{\# \# \# ~}$ \\
\hline \multirow{3}{*}{ HDL-C } & 0 Weeks & $1.58 \pm 0.16$ & $1.62 \pm 0.15$ & $1.68 \pm 0.13$ & $1.71 \pm 0.25$ & $1.69 \pm 0.19$ \\
\hline & 2Weeks & $1.61 \pm 0.21$ & $2.13 \pm 0.11^{* *}$ & $1.98 \pm 0.31$ & $2.03 \pm 0.32$ & $1.91 \pm 0.36^{\#}$ \\
\hline & 4Weeks & $1.82 \pm 0.14$ & $3.07 \pm 0.13^{* * *}$ & $2.43 \pm 0.22^{\# \#}$ & $2.65 \pm 0.31^{\#}$ & $2.21 \pm 0.27^{\# \# \# ~}$ \\
\hline
\end{tabular}

Note: Comparison of odel group and the normal group: * $\mathrm{P}<0.05$, ** $\mathrm{P}<0.01$, *** $\mathrm{P}<0.001$; comparison of knotweed group, aerobic exercise group, Polygonum combined with aerobic exercise group: \# $\mathrm{P}<0.05$, \# \# $\mathrm{P}<0.01$, \# \# \# $\mathrm{P}<0.001$. (the next table is the same ).

Table2 Different groups in the comparison of serum SOD, GSH-Px, CAT and MDA

\begin{tabular}{cccccc}
\hline Index & Control & Model control & PC & $\begin{array}{c}\text { Aerobic } \\
\text { exercise }\end{array}$ & $\begin{array}{c}\text { PC+ Aerobic } \\
\text { exercise }\end{array}$ \\
\hline SOD & $97.9 \pm 16.8$ & $69.4 \pm 14.6^{* *}$ & $89.1 \pm 17.5^{\#}$ & $79.6 \pm 17.8$ & $92.4 \pm 19.5^{\#}$ \\
GSH-Px & $13.5 \pm 1.9$ & $8.9 \pm 2.4^{* * *}$ & $12.8 \pm 2.5^{\# \#}$ & $11.9 \pm 2.7^{\# \#}$ & $13.1 \pm 3.2^{\# \#}$ \\
CAT & $49.8 \pm 5.4$ & $43.3 \pm 5.2^{*}$ & $47.9 \pm 4.7$ & $45.9 \pm 1.7$ & $49.41 \pm 5.1^{\#}$ \\
MDA $^{-1}$ & $3.54 \pm 1.2$ & $5.76 \pm 1.3^{* *}$ & $4.21 \pm 1.2^{\#}$ & $4.79 \pm 1.7$ & $4.21 \pm 1.1^{\#}$ \\
\hline
\end{tabular}




\section{Discussion and Analysis}

In recent years, clinical and experimental research of Chinese medicine for the treatment of hyperlipidemia has accumulated rich experience and great progress has been made in finding medicines that can effectively lower the body fat, such as saponins, flavonoids, alkaloids, anthraquinone, oils and fats, etc[4]. There are many reports on how Polygonum cuspidatum can regulate blood lipids. Studies have reported that different doses of resveratrol glycosides can significantly reduce TC, TG, HDL-C, LDL-C, MDA, NO levels of hyperlipidemia rats and increased serum levels of SOD in rats with hyperlipidemia, indicating that polydatin can effectively regulate blood lipid metabolism and metabolic disorders corrective radicals, thus playing a role in anti-atherosclerosis. Longtao Liu has have confirmed that the polydatin high-resolution ultrasound treatment group shows a significant reduction in the total number of plaques, and can significantly reduce serum protein expression -1 (MMP-1) and MMP-1 / tissue inhibitor of metalloproteinase-1 (TIMP-1) levels. This shows that knotweed has antioxidant effects, its mechanism of action may be related to increasing SOD, GSH-Px activity, removing toxic oxygen ions and preventing its free radical chain reactions caused by inhibition of lipid peroxidation and so on [5]. This study found that knotweed can reduce serum TG, TC, LDL-C, increases HDL-C, which is consistent with literature reports.

This study shows that knotweed can improve the body's SOD and GSH-PX activity, and inhibit the production of MDA to accelerate its clearing speed, thus effectively preventing lipid peroxidation in the body and improving the progress of lipid metabolism disorders. The possible mechanism is that knotweed can promote intestinal peristalsis and accelerate the excretion of cholesterol, reducing cholesterol absorption, which play a lowering blood pressure, lowering the quality of metabolic disorders. Another possibility is that Polygonum catecholamines can reduce capillary permeability, increased density of the endothelium, restrict access to harmful substances, thereby reducing blood viscosity, reduce the deposition of lipids. The knotweed contains anthraquinone derivatives substances, with lowering blood pressure, vasodilator effects [6]. This study shows that the effect of simple aerobic exercise on GSH-PX is obvious, without reducing serum MDA and improving the vitality of SOD and CAT. Single Polygonum cuspidatum extract can only increase SOD, GSH-Px and lower MAD content, but has no significant change in the CAT. However, a combination of aerobic exercise can increase knotweed Serum SOD, GSH-Px and CAT levels, while lowering serum MDA levels. Knotweed combined with works better in improving the body's fat metabolism than single knotweed or aerobic exercise. Combining drugs with aerobic exercise may be an effective way to treat dyslipidemia.

\section{Summary}

Both knotweed and Polygonum cuspidatum combined with aerobic exercise can effectively regulate the body's fat metabolism and reduce serum TC, TG, LDL-C levels and rise HDL-C levels. The effect of the combination of knotweed and aerobic exercise is better than single knotweed or unilateral action; the mechanism of how the combination mentioned above improves lipid metabolism disorders may relate to the inhibition of lipid abnormalities, raising serum SOD, GSH-Px levels, CAT reducing serum MDA levels and activity.

\section{References}

[1] Yan ZH, Science of Chinese Materia Medica, third ed., People's Medical Publishing House, Bei Jing,2006.

[2] Fan HT, Ding SL, Pharmacological of Polygoni Cuspidati Rhizoma, China journal of Chinese material medica. 38(2013)2545-2548. 
[3] ZHANG ST , WANG W, Myocardial protective effects of polydatin on isolated rat hearts suffering, Chin Heart J. 22(2010)355-358.

[4]Sharma S, Chopra K, Kulkami s K, Resveratrol and curcu. min suppress immune response through CD28 / CTLA-4 and CD80co-stimulatory pathway, Clin Exp Immunol. 147(2007)155-158.

[5] Lee M K, Kang S J, Poncz M, Resveratrol protects SHSY5Yneuroblastoma cells from apoptosis induced by dopamine, Exp Mol Med. 39(2007)376-369.

[6]Kariko K, Weissman D, Welsh FA, Inhibition of toll-like receptor and cytokine signaling-a unifying theme in ischemic tolerance, J Cereb Blood Flow Metab. 24(2004) 1288-1304. 